First publ. in: Bioluminescence and Chemiluminescence / ed. by Marlene A. DeLuca ... New York: Acad. Pr., 1981, pp. 97-102

\title{
FLUORESCENCE PROPERTIES OF LUCIFERASE PEROXYFLAVINS PREPARED WITH ISO-FMN AND 2-THIO FMN ${ }^{1}$
}

J. W. Hastings

The Biological Laboratories

Harvard University

Cambridge, Massachusetts

Sandro Ghisla, Manfred Kurfúrst, Peter Hemmerich

Fachbereich Biologie

Universität Konstanz

Konstanz, West Germany

The light emitting reaction catalyzed by luciferase obtained from luminous bacteria is an interesting one, and apparently quite different from several other bioluminescent reactions, such as firefly and Cypridina $(1,2)$. The overall reaction involves the mixed function oxidation of $\mathrm{FMNH}_{2}$ and long chain aldehyde by molecular oxygen, but already in the early studies (3) it was shown that the reaction could be divided into steps, the first involving the reaction of $\mathrm{O}_{2}$ with reduced flavin and the second the oxidation of the aldehyde by the intermediate.

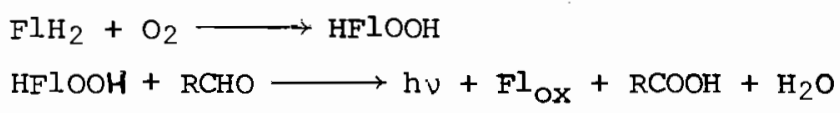

The intermediate has been isolated and characterized as a luciferase-bound peroxyflavin (4-8). A key question concerns the reaction mechanism of this intermediate with aldehyde, involving the formation of the excited molecular species responsible for light emission. In this abstract, we summarize knowledge and recent experiments relating to the identity

${ }^{1}$ These studies were supported in part by grants from the U.S. National Science Foundation (PCM 77-19917) and the Deutsche Forschungsgemeinshaft to S.G. J.W.H. was an awardee of the Alexander Van Humboldt Foundation. 


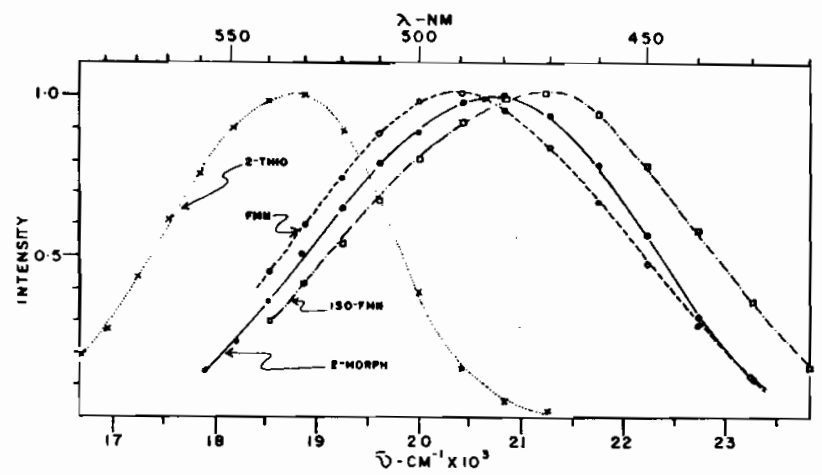

FIGURE $I$. Bioluminescence emission spectra for FMN, iso-FMN, 2-morpholino-, and 2-thiofMN. Intensity values are normalized and plotted in the terms of wave numbers (from 9).

of the emitting species in the reaction.

Since FMN occurs as a product of the reaction, it had seemed at first that its excited singlet might be designated as the emitting species. But the fact that its fluorescence emission is centered at about $525 \mathrm{~nm}$ while that of the in vitro bacterial bioluminescence is maximal at about $490 \mathrm{~nm}$ makes this unlikely (3). In work explicitly concerned with this question, Mitchell and Hastings (9) considered several possibilities. One was that the emission derives from an enzyme-bound excited state of FMN, with the blue shift attributed to the protein environment. A second possibility was that a flavin chemically altered in the course of the reaction was the species responsible for light emission. A third was that the emission results from some luciferase-bound or associated group, even perhaps an aromatic amino acid residue of the luciferase itself (10).

In order to evalute these possibilities we tested a considerable number of flavin isomers and analogues for activity; several were found to exhibit low but authentic activity (9). Since these isomers differ from FMN itself with regard to the wavelength of fluorescence emission, it was of interest to determine if the color of the bioluminescence was similarly changed. Changes were indeed observed (Fig. 1), but they did not correlate with the excited singlet fluorescence of the flavin as such (Table I). The possibility that the flavin cation might be the emitting species was also suggested (11), and although its fluorescence emission correlates with bioluminescence in the case of FMN and possibly 2-thioFMN, it does not in the case of isoFMN (Table I). 
TABLE I. Color of Bioluminescence and Fluorescence Emissions with Different Flavins

Emission Peak wavelength (nm)

FMN ISOFMN 2-thiOFMN

\begin{tabular}{|c|c|c|c|}
\hline Bioluminescence with luciferasea & 490 & 472 & 534 \\
\hline Fluorescence of flavin in $\mathrm{H}_{2} \mathrm{O}^{a}$ & 525 & 543 & nonfluor. \\
\hline $\begin{array}{l}\text { Fluorescence of the flavin } \\
\text { cation b }\end{array}$ & $480-493$ & 530 & $510-530$ \\
\hline $\begin{array}{c}\text { Fluorescence of the luciferase- } \\
\text { peroxyflavin intermediatec }\end{array}$ & 490 & $465-470$ & $540-545$ \\
\hline
\end{tabular}

arom (9)

$b_{\text {from (i1) }}$

Cuncorrected values, Perkin Elmer MPF44

The fact that the emission spectrum of bioluminescence differed with different flavins implicates a flavin as the emitter in the purified system. Such a result would not be expected if (as in the third alternative above) some other molecule were serving as the emitter and the flavin functioned only as a reductant, since altering the flavin should not alter the emission spectrum.

Nevertheless, the possibility that flavin is not the emitter in the purified in vitro system continues to be seriously considered (12), at least in part because of the reported occurrence of secondary emitters in the in vivo mission of at least some species and strains of luminous sacteria. The proposal that in vivo bacterial "bioluminescence might ... (involve) transfer of reaction energy to a pigment adsorbed on the luciferase ..." was first put forward by van der Burg (quoted in 13), who also showed that emission spectra differ in different species (14). In addition to the "blue shifted" emission of Photobacterium phosphoreum, there is the recently discovered "yellow" emitting strain of $P$. fischeri (15). Both of these cases have now been attributed to the in vivo occurrence of secondary emitters involving prosthetic fluorescent groups attached to a protein separable in vitro from the luciferases $(16,17)$. In the case of $P$. phosphoreum the emitter has been reported to be a pteridine (18), while in $P$. fischeri it is a flavin (19). These molecules may be designated as secondary 
emitters; the purified luciferases emit (reportedly with lower quantum yields) in the absence of such secondary emitters, and at wave lengths which are characteristic of the particular flavin used to initiate the reaction.

The luciferase itself may also be determinant with regard to color. This was clearly shown by the fact that in cells possessing luciferase altered by mutation, the color of the light in vivo was red-shifted, and that the reaction catalyzed by luciferase purified from these cells was similarly color shifted (20).

Thus the luciferase-flavin intermediate should evidently be designated as the primary emitter in the pure in vitro system. What then is its structure? The experiments described below support the postulate that the emitter is an altered flavin generated as a reaction intermediate.

The flavin hydroperoxide formed in the first step of the reaction has an interesting property; its fluorescence close matches that of the bioluminescence (Fig. 2) (6). But since this intermediate occurs prior to the step responsible for populating the excited state, it cannot be the enitter as such. Its structure might, however, be a clue to that of the emitter. For example the emitter could be a flavin having some other substituent in the 4 a position, possibly the $4 a-$ hydroxy species (Fig. 3), which would be expected to have the same fluorescence properties.

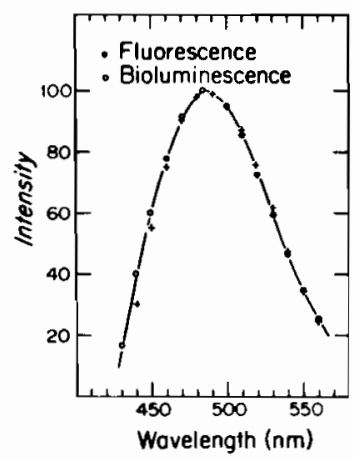

FIGURE 2. Emission spectrum of bioluminescence (o), measured directly from the cuvet during warming of a luciferase-peroxy FMN preparation in the presence of aldehyde, plotted together with the fluorescence emission spectrum of the peroxy FMN (*). Ordinate, intensity of bioluminescence and fluorescence, normalized at the peak (from 6). 


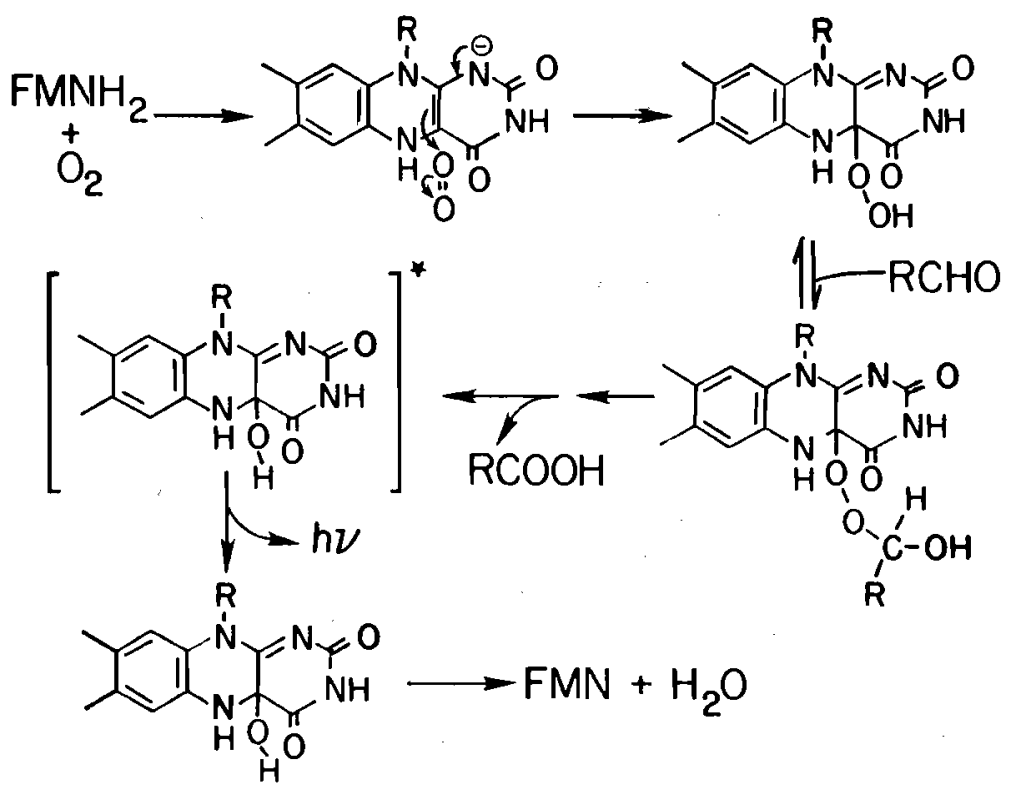

mediates, including the emitting flavin chromophore in bacterial bioluminescence (from 1).

To test this idea we examined the fluorescence of the luciferase peroxy adducts using FMN, isoFMN and 2-thioFMN. Luciferase and flavin were mixed at $0^{\circ}$, reduced by dithionite, and chromatographed on Sephadex G-25 at $2^{\circ}$ in the presence of $5 \mathrm{HM}$ tetradecanol, which stabilizes the intermediates without altering fluorescence properties (21). The fluorescence mission spectra of these adducts were found to peak at wavelengths similar to the bioluminescence emissions with the same flavins (Table I), supporting the postulate that the primary emitter in luminescence with pure bacterial luciferase is a 4a substituted flavin formed as an intermediate in the reaction. 


\section{REFERENCES}

1. Hastings, J. W., and K. H. Nealson, Ann. Rev. Microbiol. 31, 549 (1977).

2. Hastings, J. W., Ciba Foundation Symposium 31, 125 (1975).

3. Hastings, J. W., and Q. H. Gibson, J. Biol. Chem. 238, 2537 (I963).

4. Hastings, J. W., C. Balny, C. Le Peuch, and P. Douzou, Proc. Nat. Acad. SCi. 70, 3468 (1973).

5. Hastings, J. W., and C. Balny, J. Biol. Chem. 250, 7288 (1975).

6. Balny, C., and J.W. Hastings, Biochemistry 14, 4719 (1975).

7. Ghisla, S., J.W. Hastings, V. Favaudon, and J. M. Ihoste, Proc. Nat. Acad. Sci. 75, 5860 (1978).

8. Becvar, J. E.,S.-C. Tu, and J. W. Hastings, Biochemistry 17,1807 (1978).

9. Mitchell, G., and J. W. Hastings, J. Biol. Chem. 244, 2572 (1969).

10. Cormier, M. J., and J. R. Totter, Annu. Rev. Biochem. 33, 431 (1964).

11. Eley, E., J. Lee, J. M. Ihoste, C. Y. Lee, M. J. Cormier, and P. Hemmerich, Biochemistry 14, 2902 (1970).

12. Hart, R. C., and M. J. Cormier, Photochem. Photobiol. 29, 209 (1979).

13. Harvey, E. N., "Bioluminescence," p. 88. Academic Press, N.Y. (1952).

14. Spruit-van der Burg, A., Biochem. Biophys. Acta $\underline{5}, 175$ (1950).

15. Ruby, E. G., and K. H. Nealson, Science 196, 432 (1977).

16. Gast, R., J. Nearing, and K. H. Lee, Biochem. Biophys. Res. Commun. 80, 14 (1978).

17. Leisman, G., and Nealson, unpublished.

18. Koka, P., and J. Lee, Proc. Nat. Acad. Sci. 76, 3068 (1979) .

19. Leisman, G., K.H. Nealson, and J.W. Hastings, unpublished.

20. Cline, T.W., and J.W. Hastings, J. Biol. Chem. 249, 4668 (1974).

21. Tu, S.-C., Biochemistry 18, 5940 (1979). 